Check for updates

Cite this: Phys. Chem. Chem. Phys., 2017, 19, 28507

Received 29th August 2017, Accepted 2nd October 2017

DOI: $10.1039 / c 7 c p 05897 g$

rsc.li/pccp

\section{LK peptide side chain dynamics at interfaces are independent of secondary structure $\dagger$}

\author{
Michael A. Donovan, (D) ${ }^{a}$ Helmut Lutz, ${ }^{a}$ Yeneneh Y. Yimer, ${ }^{b}$ Jim Pfaendtner, (D) ${ }^{b}$ \\ Mischa Bonn (iD a and Tobias Weidner (D) *abc
}

\begin{abstract}
Protein side chain dynamics are critical for specific protein binding to surfaces and protein-driven surface manipulation. At the same time, it is highly challenging to probe side chain motions specifically at interfaces. One important open question is the degree to which the motions of side chains are dictated by local protein folding or by interactions with the surface. Here, we present a real-time measurement of the orientational dynamics of leucine side chains within leucine-lysine (LK) model peptides at the water-air interface, with three representative peptide folds: $\alpha$-helix, $3_{10}$-helix and $\beta$-strand. The results, modeled and supported by molecular dynamics simulations, show that the different peptide folds exhibit remarkably similar subpicosecond orientational side chain dynamics at the air/water interface. This demonstrates that the side chain motional dynamics is decoupled from the local secondary structure.
\end{abstract}

Proteins are highly dynamic entities, and protein dynamics have direct implications for protein structure and function. ${ }^{1,2}$ Side chains play a role in protein driven surface manipulation, ${ }^{3}$ enzymatic catalysis, ${ }^{4,5}$ and in protein-protein interactions. ${ }^{6}$ In addition, they undergo rotameric transitions ${ }^{7}$ and perform torsional motions on pico- to nanosecond timescales. ${ }^{1,8-10}$ Protein side chain dynamics are typically determined via NMR spectroscopy through linewidth measurements where changes in conformational entropy can be inferred.,11-19 NMR can track changes in a protein's chemical environment upon complexation and has been used to show that, in solution, side chain dynamics of methyl containing residues can provide insights about different local environments within a protein. ${ }^{6}$ The dynamics are not monitored directly in real time, however, but they are inferred from spectral line shapes. ${ }^{11,20}$

\footnotetext{
${ }^{a}$ Max Planck Institute for Polymer Research, Ackermannweg 10, 55128 Mainz, Germany

${ }^{b}$ Department of Chemical Engineering, University of Washington, 105 Benson Hall, Seattle, WA 98195, USA

${ }^{c}$ Department of Chemistry, Aarhus University, Langelandsgade 140, 8000 Aarhus C, Denmark. E-mail: weidner@chem.au.dk

$\dagger$ Electronic supplementary information (ESI) available: Experimental details, simulation details, and additional modelling. See DOI: $10.1039 / \mathrm{c} 7 \mathrm{cp} 05897 \mathrm{~g}$
}

An advantage of electronic ${ }^{22-24}$ and vibrational ${ }^{25,26}$ spectro- $^{-}$ scopic methods is that they can directly resolve sub-picosecond events and thereby provide direct insight into how protein side chains move.

Specifically at interfaces, information about protein side chain dynamics on pico-second timescales has been lacking. Yet side chain motions are particularly important here, since they mediate the contact to the interface and play a role in the recognition of hard and soft tissue. ${ }^{13,27,28}$ The understanding of these dynamic interactions can help unravel key biological processes from biomineralization, ${ }^{11,21}$ protein membrane interactions, ${ }^{29}$ host-guest interactions, ${ }^{6,30}$ to the specific protein recognition of mineral surfaces. ${ }^{31}$

One important question is the impact of local folding within proteins on the dynamics of side chains when interacting with surfaces. Here, leucine-lysine (LK) peptides provide an ideal model system. LK peptides are known to form well-defined secondary structures at hydrophobic, hydrophilic and aqueous interfaces based on their hydrophobic periodicity (Scheme 1). ${ }^{13,21,32,33}$ At the air/water interface, hydrophobic leucine side chains point toward the vapor phase whereas hydrophilic lysines are oriented towards the aqueous phase. ${ }^{21}$ In this study, we probe leucine dynamics of LKs folding into $\alpha$ helices (LK $\alpha 14$ : Ac-LKKLLK LLKKLLKL-OH), $\beta$ strands (LK $\beta 15$ : Ac-LKLKLKLKLKLKLKL$\mathrm{OH}$ ), and $3_{10}$ helices (LK3 $3_{10}$ : Ac-LLKLLKLLKLLKL-OH). These peptides have been studied extensively as model systems for protein-surface interactions and therefore provide an ideal starting point to explore interfacial protein dynamics. ${ }^{13,21,27,33-35}$

Previously, interface-specific time-resolved vibrational spectroscopy has helped unveil the orientational dynamics of a monomeric leucine amino acid at the air/water interface. It was shown that the methyl units reoriented diffusively on a time scale of $20 \mathrm{ps}$ with diffusivities of $D_{\varphi}=0.07 \mathrm{rad}^{2} \mathrm{ps}^{-1}$ in the plane of the surface and $D_{\theta}=0.05 \mathrm{rad}^{2} \mathrm{ps}^{-1}$ out of plane (see Scheme 1 for definition of angles). Here we report on how the folding of the peptide backbone affects ultrafast motions by following the dynamics of leucine side chains in LK peptides with different folds at the air/water interface using time-and 


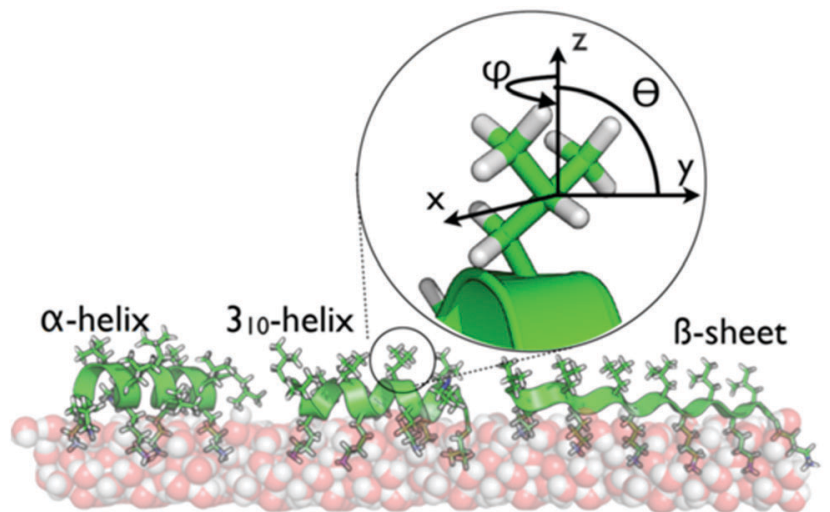

Scheme 1 The secondary structures of $L K \alpha 14, L K 3_{10}$, and $L K \beta 15$ at the air/ water interface are dictated by the hydrophobic periodicity of the amino acid sequence. The peptides have been previously shown to bind with the indicated foldings. ${ }^{21}$ Hydrophobic leucine side chains are exposed to the vapor phase while the hydrophilic lysines remain hydrated in the water phase. The inset shows the geometry of the leucine sites used in this study.

polarization-resolved sum-frequency generation spectroscopy (TPSFG). ${ }^{37-39}$ We quantify the results through the help of numerical modelling and molecular dynamics (MD) simulations.

TPSFG is a time resolved variant of surface-sensitive SFG. ${ }^{40-46}$ In TPSFG, an intense, linearly polarized IR pump pulse partially depletes the vibrational ground state within a sub-ensemble of molecules whose vibrational transition moment is oriented preferentially along the pump polarization direction. Orientational motion and vibrational energy transfer can be followed while the excited vibrational group relaxes back to equilibrium. ${ }^{37-39}$ When the pump polarization is parallel $(||)$ to that of the probe, the signal decays due to both intramolecular vibrational relaxation (IVR) and orientational motions back into the plane (ip) of the surface, whereas when the pump direction is orthogonal $(\perp)$, IVR and out of plane motions (oop) are most efficiently sampled.

To verify the interfacial secondary structure for the LK peptides, we have first recorded static ssp (s polarized SF, $s$ polarized Vis, p polarized IR) SFG spectra in the amide-I region. The spectra are shown in Fig. 1a, and display intense resonances ranging from $1642 \mathrm{~cm}^{-1}$ for LK $\alpha 14$ to $1655 \mathrm{~cm}^{-1}$ for LK $\beta 15$. These resonance positions are in agreement with published values ${ }^{47}$ for the target folds.

The time-resolved experiments focus on the leucine methyl $\mathrm{C}-\mathrm{H}$ resonances. Representative static sps (s polarized SF, p polarized VIS, s polarized IR probe) spectra of the C-H region are shown in Fig. $1 \mathrm{~b}$ for $0.1 \mathrm{mg} \mathrm{mL} \mathrm{m}^{-1}$ solutions of three different folds of LK peptide adsorbed to the air/ $\mathrm{D}_{2} \mathrm{O}$ interface. This polarization combination is chosen based on our previous study with leucine. ${ }^{36}$ Qualitatively, all three spectra show an intense resonance at $2958 \mathrm{~cm}^{-1}$ which is assigned to the methyl in-plane asymmetric (AS) stretch. ${ }^{21}$ It is this AS stretching mode we are predominantly exciting with an intense mid-IR pump pulse which spans the entire aliphatic stretching region (Fig. 1b). The pump polarization is alternated between $\mathrm{s}(\mathrm{I})$ and $\mathrm{p}(\perp)$ to generate the traces for the signal near $2958 \mathrm{~cm}^{-1}$ shown in Fig. 2. The data in Fig. 2 show the ground state bleach

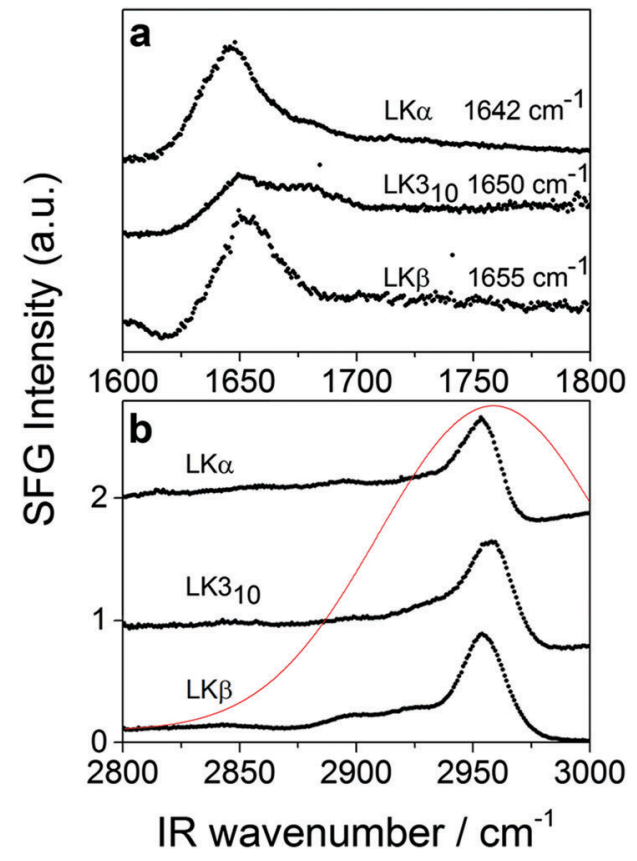

Fig. 1 Static SFG spectra of LK $\alpha 14$, LK310, and LK $\beta 15$ adsorbed to the air/ $\mathrm{D}_{2} \mathrm{O}$ interface (a) amide-I region using ssp polarization (see text for details) (b) using sps polarization combination in the aliphatic stretching region (see text for details). The profile of the excitation pulse is shown in red.

of the methyl AS stretch mode around zero delay between pump and probe followed by subsequent recovery of the signal. The solid lines which follow the recovery of the signal are numerically simulated according to reference ${ }^{37}$ and will be discussed later in the text.

For a first approximation of the relaxation times, we can fit the signal recovery rates $k_{(\|, \perp)}=1 / \tau_{(\|, \perp)}$ with exponential kinetics (see ESI $\dagger$ for fits and further discussion). The results are summarized in Table 1. Importantly, the fits show that the parallel relaxation is significantly faster compared to the perpendicular dynamics, which shows that reorientation must contribute to the measured signals. ${ }^{39}$

The polarized excitation pulse temporarily changes the symmetry at the surface and transient tensor elements add to the signal. ${ }^{37}$ This complicates the direct quantitative analysis.

In addition, the experimentally measured traces are underdetermined since the signal recovery of the transient data depends on three time constants: the vibrational relaxation time $T_{1}$, the in-plane diffusivity $D_{\varphi}$, and the out-of-plane diffusivity $D_{\theta}$.

In the limiting case for small angular spread $\Delta \theta$ (i.e. $\Delta \theta \ll$ $\sin \theta_{0}$ ) and negligible out of plane motion, a convenient way to determine the molecular diffusion directly is to analyze the anisotropy decay (see ESI $\dagger$ for details)..$^{37,48}$ The in plane diffusivity $D_{\varphi}$ can, in this limiting case, be determined directly by plotting the anisotropy decay between the parallel and perpendicularly pumped traces. ${ }^{37}$ However, since the present peptide systems allow a broad distribution of side chain conformations and out-of-plane motion it is not a priori apparent that this would be a reliable approach here. 


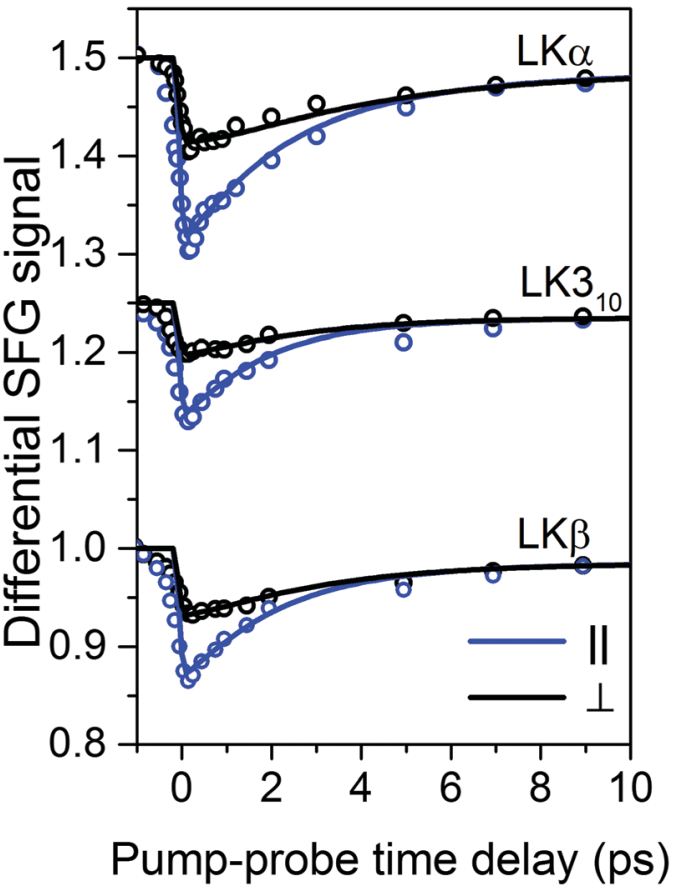

Fig. 2 Transient SFG traces of $L K \alpha 14$, LK310, and LK $\beta 15$ adsorbed to the air/ $\mathrm{D}_{2} \mathrm{O}$ interface: experimental pump-probe transients of $\mathrm{LK}$ peptides adsorbed to the air $/ \mathrm{D}_{2} \mathrm{O}$ interface (open circles) along with numerically simulated orientation-dependent dynamic response (solid lines). The corresponding inferred diffusion coefficients $D_{\theta}$ and $D_{\varphi}$ are summarized in Table 1. Traces are offset for clarity.

Table 1 Vibrational time constants, diffusion rate coefficients and tilt angle information. Error margins are given in parenthesis

\begin{tabular}{|c|c|c|c|c|c|c|}
\hline Peptide & $\begin{array}{l}\tau_{1, \|} \\
(\mathrm{ps})\end{array}$ & $\begin{array}{l}\tau_{1, \perp} \\
\text { (ps) }\end{array}$ & $\begin{array}{l}T_{1}^{\operatorname{sim}} \\
(\mathrm{ps})\end{array}$ & $\begin{array}{l}D_{\varphi} \\
\left(\mathrm{rad}^{2} \mathrm{ps}^{-1}\right)\end{array}$ & $\begin{array}{l}D_{\theta} \\
\left(\operatorname{rad}^{2} \mathrm{ps}^{-1}\right)\end{array}$ & $\begin{array}{l}\theta_{0} \\
\text { (degree) }\end{array}$ \\
\hline $\mathrm{LK} \alpha 14$ & $\begin{array}{l}2.99 \\
(0.24)\end{array}$ & $\begin{array}{l}4.89 \\
(0.71)\end{array}$ & 3.3 & $\begin{array}{l}0.12 \\
(0.04)\end{array}$ & $\begin{array}{l}0.06 \\
(0.01)\end{array}$ & 62 \\
\hline LK $\beta 15$ & $\begin{array}{l}2.43 \\
(0.29)\end{array}$ & $\begin{array}{l}5.57 \\
(0.59)\end{array}$ & 2.5 & $\begin{array}{l}0.13 \\
(0.02)\end{array}$ & $\begin{array}{l}0.04 \\
(0.01)\end{array}$ & 62 \\
\hline $\mathrm{LK} 3_{10}$ & $\begin{array}{l}2.08 \\
(0.27)\end{array}$ & $\begin{array}{l}3.63 \\
(0.58)\end{array}$ & 2.2 & $\begin{array}{l}0.13 \\
(0.02)\end{array}$ & $\begin{array}{l}0.05 \\
(0.01)\end{array}$ & 63 \\
\hline
\end{tabular}

We therefore performed molecular dynamics (MD) simulations to track the peptide dynamics and to combine the experimental data with theoretical transients calculated from the MD data.
The combination of MD and time resolved SFG has recently been successfully applied to monomeric leucine adsorbed at the air-water interface. ${ }^{36}$ The method uses MD simulation of the interfacial LK peptide dynamics as a basis for a numerical model $^{37}$ to calculate the time-resolved SFG response. The numerical model requires information about (i) the static molecular orientation, defined by the mean tilt angle $\theta_{0}$ and the angular spread $\Delta \theta$; (ii) the orientational in plane and out of plane diffusivities $\left(D_{\varphi}, D_{\theta}\right)$; and (iii) the vibrational relaxation time $T_{1}$.

To computationally determine the molecular orientation and the reorientational diffusion of the methyl groups of leucine at the vacuum/water interface, we performed all-atom MD simulations at $298 \mathrm{~K}$. Three separate simulations were performed for a layer of 23 molecules of LK $\alpha 14$, LK $\beta 15$, and $\mathrm{LK}_{10}$. Further details about the simulations are presented in the ESI. $\dagger$ Briefly, the peptides were packed as a layer on 8 by $8 \mathrm{~nm}$ in between a $6.8 \mathrm{~nm}$ thick slab of water and $7 \mathrm{~nm}$ of vacuum. To approximate experimental conditions, phosphate ions were added, and the box was neutralized by adding chloride ions. Note that while the simulations can provide, $\theta_{0}, \Delta \theta, D_{\varphi}$ and $D_{\theta}$, it cannot provide $T_{1}$. Table 1 lists guessed values of $T_{1}$ defined as $T_{1}{ }^{\text {sim }}$ which are arrived at by finding the best visual fit to the data (see $\mathrm{ESI} \dagger$ Section IV). $T_{1}$ values determined experimentally are labelled $T_{1}$ and found in ESI $\dagger$ Table S1.

After an equilibration period of $100 \mathrm{~ns}, 5 \mathrm{~ns}$ were simulated with coordinates being written to a trajectory file with a frequency of $4 \mathrm{fs}$ for analysis. As expected for a peptide layer with random in-plane orientation, the azimuthal $\varphi$ angles showed no predominant orientation for all peptides (see ESI $\dagger$ ). The mean tilt angle $\theta_{0}$ and the angular spread $\Delta \theta$ were obtained from a Gaussian fit (see ESI $\dagger$ for details) to the methyl group distributions shown in Fig. 3 . The mean tilt angles $\theta_{0}$ for the respective $\alpha, \beta$, and $3_{10}$ folds where $62^{\circ}, 62^{\circ}$, and $63^{\circ}$ with angular spreads $\Delta \theta$ of $32^{\circ}, 39^{\circ}$, and $29^{\circ}$, respectively. This shows that the assumption of the limiting case of only in-plane reorientation is not applicable. The in- and out-of-plane methyl reorientational diffusion coefficients $D_{\varphi}$ and $D_{\theta}$ were determined by first numerically solving the two-dimensional rotational diffusion equation for a specific set of $\left(D_{\varphi}, D_{\theta}\right)$. Then the square of residuals, $\chi^{2}$, between the thus calculated results and the simulation derived
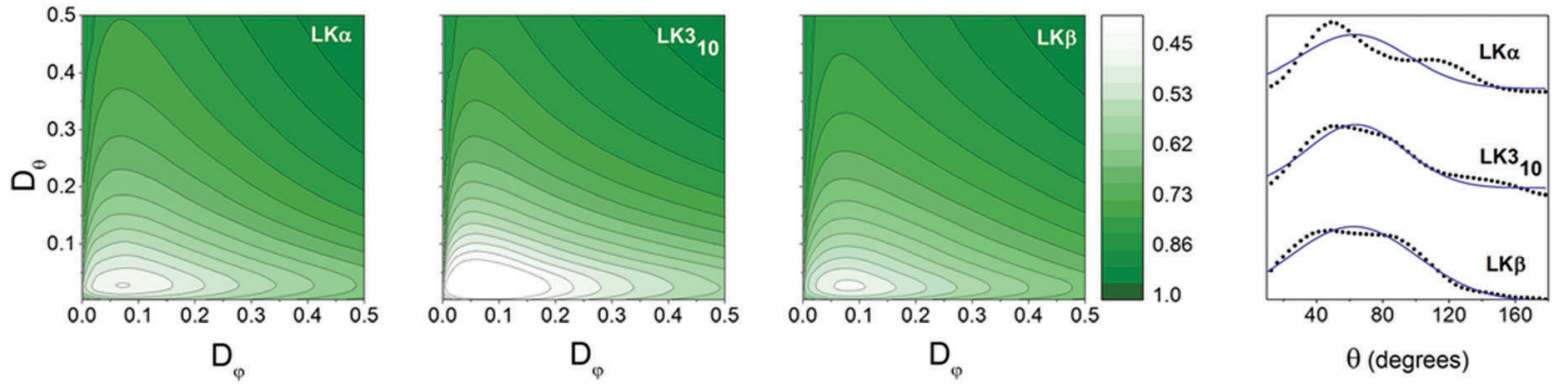

Fig. 3 Orientational information: contour plots: variation of $\chi^{2}$ with $D_{\varphi}$ and $D_{\theta}$ for methyl groups of LK peptides at the air-water interface. $D_{\varphi}$ and $D_{\theta}$ are inferred from the point for which the variance $\chi^{2}$ is at a minimum. Values for all three peptides may be found in Table 1. Far right: Orientation distributions of leucine methyl groups in LK peptides at the air/water interface in black. Gaussian fits are shown with blue line. 
time-dependent methyl group angle dependent (i.e. $f(\theta, \varphi)$ ) population distributions was calculated (Fig. 3b see ESI $\dagger$ for details). We determined that $\chi^{2}$ for $\alpha$, and $\beta$, and $3_{10}$ peptides exhibit minima for the out-of-plane diffusion $D_{\theta}$ at respectively $0.06 \pm 0.01,0.04 \pm 0.01$, and $0.05 \pm 0.01 \mathrm{rad}^{2} \mathrm{ps}^{-1}$ and for the in-plane diffusion $D_{\varphi}$ at $0.12 \pm 0.04,0.13 \pm 0.02$, and $0.13 \pm$ $0.02 \mathrm{rad}^{2} \mathrm{ps}^{-1}$ (Fig. 3a-c). The simulations thus indicate that leucine methyl groups reorient - on average - at twice the rate in-plane than out-of-plane, but with no significant differences between the respective folds.

The vibrational relaxation times $T_{1}^{\mathrm{sim}}$ used for the numerical simulation $^{37}$ to produce the traces in Fig. 2 were initially approximated to be close to previously reported values of $\sim 3$ ps for methyl stretching vibrations of long aliphatic chains; ${ }^{26,38,49}$ precise values were subsequently obtained by choosing vibrational relaxation times which give the best visual fit to the experimental data. It is interesting to note, that the $\alpha$-helix motif showed a slower vibrational relaxation compared to the $\beta$-strand and $3_{10}$-helix. This is, in line with reports by Backus and Stock et al., who have shown that structural flexibility within peptides in solution enhances vibrational energy transfer. ${ }^{50,51}$ Plotting the number of peptide-peptide hydrogen bonds (see ESI $\dagger$ ) showed that $\mathrm{LK} \alpha 14$ has indeed the largest number of $\mathrm{H}$-bonds.

The simulated transient SFG responses for the different peptide folds with orientational parameters obtained from MD are shown as solid lines in Fig. 2. The obtained theoretical traces capture the signal depletion and recovery in the experimental data very well. The close match between theory and experiment confirms the peptide side chain dynamics obtained from simulation and shows that the methyl reorientation dynamics are, within the error margins, independent of the folding of the peptide backbone.

It is interesting to compare dynamics of the leucine side chains within peptides monomeric leucines at the air-water interface. The out of plane dynamics are very similar for the peptide-bound leucine as compared to monomeric leucine, with a diffusivity of $c a .0 .06 \mathrm{rad}^{2} \mathrm{ps}^{-1}$ for the peptides and $0.05 \mathrm{rad}^{2} \mathrm{ps}^{-1}$ for the monomers. The in plane diffusivity, however, is sped up by a factor of two with an average diffusivity of $0.12 \mathrm{rad}^{2} \mathrm{ps}^{-1}$ for the peptides and $0.07 \mathrm{rad}^{2} \mathrm{ps}^{-1}$ for the isolated leucine. Likely, the binding geometry and lateral proximity between neighbouring leucines enforced by the peptide backbone leads to more rapid motion within the surface plane. Going to much larger proteins, the reorientational time scales found for the LKs are comparable with methyl groups within the hydrophobic core of globular proteins (5-80 ps) as determined with NMR. ${ }^{12}$

\section{Conclusions}

Our data show that at the interface the side chain orientational dynamics are independent of the backbone folding. This is surprising in view of the diversity of steric environments side chain groups experience within helical and strand-like folding motifs within peptide monolayers. This independence of backbone structure also means that large, complex proteins with unknown interfacial folding can be studied using time-resolved SFG. While the present study uses model peptides and can only provide a first view of the role of folding for side chain dynamics, the study is a first step understanding this aspect of functional dynamics beyond the traditional structure-function paradigm.

\section{Conflicts of interest}

There are no conflicts to declare.

\section{Acknowledgements}

M. D. acknowledges financial support from the International Max Planck Research School, Cho-Shuen Hsieh, Johannes Franz, and Ruth Livingstone for discussion and for providing the Matlab code to analyze experimental data. T. W. thanks the Deutsche Forschungsgemeinschaft (WE 4478/4-1) and the Aarhus University Research Foundation (AUFF). J. P. acknowledges support from NSF award CBET-1264459 and the Hyak supercomputer system supported in part by UW-IT. Open Access funding provided by the Max Planck Society.

\section{Notes and references}

1 J. R. Lewandowski, M. E. Halse, M. Blackledge and L. Emsley, Science, 2015, 348, 578-581.

2 A. B. Law, P. J. Sapienza, J. Zhang, X. Zuo and C. M. Petit, J. Am. Chem. Soc., 2017, 139, 3599-3602.

3 T. Weidner, M. Dubey, N. F. Breen, J. Ash, J. E. Baio, C. Jaye, D. A. Fischer, G. P. Drobny and D. G. Castner, J. Am. Chem. Soc., 2012, 134, 8750-8753.

4 K. E. Bowers and C. A. Fierke, Biochemistry, 2004, 43, 5256-5265.

5 Z. Wang, C. E. Bertrand, W.-S. Chiang, E. Fratini, P. Baglioni, A. Alatas, E. E. Alp and S.-H. Chen, J. Phys. Chem. B, 2013, 117, 1186-1195.

6 A. L. Lee, S. A. Kinnear and A. J. Wand, Nat. Struct. Mol. Biol., 2000, 7, 72-77.

7 P. Schanda, M. Huber, J. Boisbouvier, B. H. Meier and M. Ernst, Angew. Chem., Int. Ed., 2011, 50, 11005-11009.

8 K. A. Henzler-Wildman, M. Lei, V. Thai, S. J. Kerns, M. Karplus and D. Kern, Nature, 2007, 450, 913-916.

9 O. F. Lange, N.-A. Lakomek, C. Farès, G. F. Schröder, K. F. A. Walter, S. Becker, J. Meiler, H. Grubmüller, C. Griesinger and B. L. de Groot, Science, 2008, 320, 1471.

10 R. G. Smock and L. M. Gierasch, Science, 2009, 324, 198.

11 R. Adrienne, A. Jason, Z. Ariel, L. M. David, J. G. Jeffrey, G. Gil and D. Gary, Proteins at Interfaces III State of the Art, American Chemical Society, 2012, vol. 1120, ch. 4, pp. 77-96.

12 L. K. Nicholson, L. E. Kay, D. M. Baldisseri, J. Arango, P. E. Young, A. Bax and D. A. Torchia, Biochemistry, 1992, 31, 5253-5263.

13 T. Weidner, N. F. Breen, K. Li, G. P. Drobny and D. G. Castner, Proc. Natl. Acad. Sci. U. S. A., 2010, 107, 13288-13293.

14 L. Vugmeyster, D. Ostrovsky, T. Villafranca, J. Sharp, W. Xu, A. S. Lipton, G. L. Hoatson and R. L. Vold, J. Phys. Chem. B, 2015, 119, 14892-14904. 
15 Y. Fu, V. Kasinath, V. R. Moorman, N. V. Nucci, V. J. Hilser and A. J. Wand, J. Am. Chem. Soc., 2012, 134, 8543-8550.

16 T. Rajitha Rajeshwar, J. C. Smith and M. Krishnan, J. Am. Chem. Soc., 2014, 136, 8590-8605.

17 G. P. Lisi and J. P. Loria, Chem. Rev., 2016, 116, 6323-6369.

18 T. I. Igumenova, K. K. Frederick and A. J. Wand, Chem. Rev., 2006, 106, 1672-1699.

19 L. Vugmeyster, D. Ostrovsky, M. A. Clark, I. B. Falconer, G. L. Hoatson and W. Qiang, Biophys. J., 2016, 111, 2135-2148.

20 L. Columbus and W. L. Hubbell, Trends Biochem. Sci., 2002, 27, 288-295.

21 H. Lutz, V. Jaeger, R. Berger, M. Bonn, J. Pfaendtner and T. Weidner, Adv. Mater. Interfaces, 2015, 2, 1500282.

22 J. Bredenbeck, J. Helbing, J. R. Kumita, G. A. Woolley and P. Hamm, Proc. Natl. Acad. Sci. U. S. A., 2005, 102, 2379-2384.

23 Y. Qin, L. Wang and D. Zhong, Proc. Natl. Acad. Sci. U. S. A., 2016, 113, 8424-8429.

24 G. Zhang, J. Li, P. Cui, T. Wang, J. Jiang and O. V. Prezhdo, J. Phys. Chem. Lett., 2017, 1031-1037, DOI: 10.1021/ acs.jpclett.7b00311.

25 Y. S. Kim and R. M. Hochstrasser, J. Phys. Chem. B, 2009, 113, 8231-8251.

26 A. Ghosh, M. Smits, J. Bredenbeck, N. Dijkhuizen and M. Bonn, Rev. Sci. Instrum., 2008, 79, 093907.

27 T. Weidner, N. F. Breen, G. P. Drobny and D. G. Castner, J. Phys. Chem. B, 2009, 113, 15423-15426.

28 J. L. Lorieau, L. A. Day and A. E. McDermott, Proc. Natl. Acad. Sci. U. S. A., 2008, 105, 10366-10371.

29 D. K. Schach, W. Rock, J. Franz, M. Bonn, S. H. Parekh and T. Weidner, J. Am. Chem. Soc., 2015, 137, 12199-12202.

30 P. T. Corbett, J. Leclaire, L. Vial, K. R. West, J.-L. Wietor, J. K. M. Sanders and S. Otto, Chem. Rev., 2006, 106, 3652-3711.

31 Proteins at Interfaces III State of the Art, ed. T. Horbett, J. L. Brash and W. Norde, American Chemical Society, 2012, vol. 1120.

32 G. Guiffo-Soh, B. Hernández, Y.-M. Coïc, F.-Z. BoukhalfaHeniche and M. Ghomi, J. Phys. Chem. B, 2007, 111, 12563-12572.
33 S. Roy, T. L. Naka and D. K. Hore, J. Phys. Chem. C, 2013, 117, 24955-24966.

34 G. J. Holinga, R. L. York, R. M. Onorato, C. M. Thompson, N. E. Webb, A. P. Yoon and G. A. Somorjai, J. Am. Chem. Soc., 2011, 133, 6243-6253.

35 D. C. Phillips, R. L. York, O. Mermut, K. R. McCrea, R. S. Ward and G. A. Somorjai, J. Phys. Chem. C, 2007, 111, 255-261.

36 M. A. Donovan, Y. Y. Yimer, J. Pfaendtner, E. H. G. Backus, M. Bonn and T. Weidner, J. Am. Chem. Soc., 2016, 138, 5226-5229.

37 H.-K. Nienhuys and M. Bonn, J. Phys. Chem. B, 2009, 113, 7564-7573.

38 S. Yamamoto, A. Ghosh, H.-K. Nienhuys and M. Bonn, Phys. Chem. Chem. Phys., 2010, 12, 12909-12918.

39 C.-S. Hsieh, R. K. Campen, A. C. Vila Verde, P. Bolhuis, H.-K. Nienhuys and M. Bonn, Phys. Rev. Lett., 2011, 107, 116102.

40 X. D. Zhu, H. Suhr and Y. R. Shen, Phys. Rev. B: Condens. Matter Mater. Phys., 1987, 35, 3047-3050.

41 R. Lu, W. Gan, B.-H. Wu, H. Chen and H.-F. Wang, J. Phys. Chem. B, 2004, 108, 7297-7306.

42 M. Fang and S. Baldelli, J. Phys. Chem. Lett., 2015, 6, 1454-1460.

43 M. R. Watry and G. L. Richmond, J. Phys. Chem. B, 2002, 106, 12517-12523.

44 N. Ji and Y.-R. Shen, J. Chem. Phys., 2004, 120, 7107-7112.

45 M. Sovago, R. K. Campen, G. W. H. Wurpel, M. Müller, H. J. Bakker and M. Bonn, Phys. Rev. Lett., 2008, 100, 173901.

46 M. Vinaykin and A. V. Benderskii, J. Phys. Chem. B, 2013, 117, 15833-15842.

47 A. Barth and C. Zscherp, Q. Rev. Biophys., 2003, 35, 369-430. 48 D. Zimdars, J. I. Dadap, K. B. Eisenthal and T. F. Heinz, J. Phys. Chem. B, 1999, 103, 3425-3433.

49 M. Smits, A. Ghosh, J. Bredenbeck, S. Yamamoto, M. Müller and M. Bonn, New J. Phys., 2007, 9, 390.

50 E. H. G. Backus, R. Bloem, R. Pfister, A. Moretto, M. Crisma, C. Toniolo and P. Hamm, J. Phys. Chem. B, 2009, 113, 13405-13409.

51 S. Buchenberg, N. Schaudinnus and S. Gerhard, J. Chem. Theory Comput., 2015, 11, 1330-1336. 